\title{
Fonemas segmentales del rromanés hablado por los gitanos grecos de Neuquén (Argentina) *
}

\author{
Pamela H. Barría Elgueta** \\ Gastón F. Salamanca Gutiérrez***
}

\begin{abstract}
Resumen
Este estudio, dividido en dos artículos, presenta una descripción fonológica del rromanés hablado por los gitanos grecos de Neuquén, Argentina. En este primer artículo, presentamos los fonemas segmentales y las realizaciones alofónicas de esta variante, una comparación de dicho inventario con el de las variantes jorajané de Chile y rusa de Argentina, y un cuento transcrito fonética y fonémicamente. Se aprecian algunas diferencias tanto en el inventario fonémico como alofónico entre estas variantes.
\end{abstract}

Palabras clave: Gitanos, Rromanés, Gitanos de Argentina, Gitanos grecos, Fonemas segmentales.

\section{Segmental phonemes of the romany spoken by Greco gypsies from Neuquén (Argentina)}

\begin{abstract}
The present research, divided into two articles, shows a phonological description of the romany spoken by the Greco-gypsies from Nuequén, Argentina. In this first article, segmental phonemes and allophonic realization of that variety are presented. And a comparison of that inventory to the varieties: Jorajané from Chile and Russian from Argentina. Also a tale transcribed both phonetically and phonemically. We observe some differences both in the phonemic and allophonic inventory between these varieties.
\end{abstract}

Key words: Gypsies, Romani, Gypsies of Argentina, Gitanos grecos, segmental phonemes.

Recibido: 25-10-2013

Aceptado: 03-03-2013

\footnotetext{
* Este trabajo presenta los principales hallazgos de la tesis de Magíster en Lingüística Aplicada "Fonemas segmentales del rromanés hablado por los gitanos grecos de Neuquén". Para escribir las palabras en la lengua gitana, utilizamos el grafemario propuesto por Salamanca y Lizarralde (2008).

** Magíster en Lingüística Aplicada, Universidad de Concepción, Concepción, Chile. pambarria@udec.cl

*** Doctor en Lingüística, Universidad de Concepción, Concepción, Chile. gaston.salamanca@gmail.com
} 


\section{Introducción}

Los gitanos son una etnia que se encuentra presente en los cinco continentes. En América, por ejemplo, existen grupos radicados en Chile, Argentina, Perú, Bolivia, México, Uruguay y Estados Unidos.

En Latinoamérica, existen dos grupos o razas principales, denominadas por ellos mismos como rrom y ludar. Ambos se diferencian entre sí en sus costumbres, formas de vida y, principalmente, sus lenguas. Dentro del grupo rrom, existen, a su vez, subgrupos o identidades, los cuales, en algunos casos, indican el lugar de procedencia. Es el caso, por ejemplo, de los gitanos rrom rusos y grecos de Argentina.

La lengua del grupo gitano rrom es denominada por ellos mismos y los romistas ${ }^{1}$ como rromaní, rromané o rromanés, y, de acuerdo al grupo que la habla, en algunos casos se especificará, si este es un rromané ruso, greco, jorajané (turco), entre otros. Según lo manifestado por los propios gitanos rrom (tanto chilenos como argentinos), se habla una misma lengua en todo el mundo, pero con algunas diferencias; es lo que técnicamente denominamos "variaciones dialectales". Resulta relevante, en consecuencia, determinar en qué consisten específicamente estas diferencias.

En Chile existen hablantes de rromané y ludar. Los estudios del rromané se inician con las publicaciones "Gitanos de Chile, un acercamiento etnolingüístico" (Salamanca y González, 1999) y "Descripción fonológica del rromané de Chile" (González y Salamanca, 2001). Más adelante, se publican trabajos en el nivel morfológico y sintáctico; incluso, hay una propuesta de grafemario y estudios que incursionan en la vitalidad de la lengua.

Por otra parte, dos descripciones fonológicas se refieren a la lengua hablada por los gitanos rrom y ludar de Argentina (González, 1997 y Salamanca, 1997, respectivamente). Ambos estudios se realizaron en la ciudad de Buenos Aires.

Como se puede apreciar, es escaso el conocimiento que se tiene del rromané y sus distintas variantes. En consecuencia, las descripciones

1 Nombre acuñado por los especialistas para referirse a quienes investigan esta lengua.

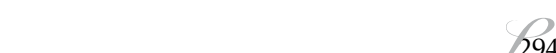


que se puedan realizar tienen un valor inicial intrínseco: conocer las simetrías y desfases entre las (pocas) variantes ya descritas y las que se seguirán describiendo; esto junto con lo que estas descripciones nos pueden informar con respecto al cambio lingüístico en general y los experimentados por la lengua de los gitanos rrom, en particular.

El presente trabajo tiene como objetivo principal presentar los fonemas segmentales de la variante conocida como rromanés greco, hablado en Neuquén. Específicamente, se determina el inventario de fonemas y alófonos de esta variante, se compara dicho inventario con las variantes jorajané de Chile y rusa de Argentina; y se propone un grafemario ad hoc, utilizando como base la propuesta para el rromané jorajané de Chile realizada por Salamanca y Lizarralde (2008). En este primer primer artículo, presentamos los resultados correspondientes a los dos primeros puntos.

\section{Antecedentes Etnográficos}

\section{Gitanos rrom en Argentina}

\subsection{Procedencia}

Aunque no se sabe la fecha exacta de ingreso de los gitanos a Argentina, se estima que ésta se produjo a mediados del siglo XIX. Fernández (2009) entrega detalles interesantes sobre los gitanos de Argentina:
Aproximadamente 300 mil Rom residen en Argentina, pertenecientes a los siguientes grupos: Kalderash griegos, moldavos y rusos, algunas familias Lovari y algunos Rom Xoraxané de Chile (todos estos grupos hablan Romaní), Kalé argentinos y españoles (ambos grupos vinieron de España en épocas diferentes y hablan sólo español) y Boyash que vinieron de Serbia y Rumania y sólo hablan rumano entre ellos.

1.2 Grupos y oficios: En Argentina, al igual que en Chile, existen dos grandes grupos de gitanos diferenciados lingüísticamente. Estos se denominan a sí mismos rrom y ludar. El grupo mayoritario, y más conservadoren cuanto a sus costumbres, es el grupo rrom. Estos distinguen entre sí subgrupos, de acuerdo al lugar desde el cual emigraron. Así, por ejemplo, los gitanos rom de Argentina mencionan como "razas" distintas a los "húngaros", "rusos", "grecos", "machuanos", "moldovanos", etc. 
El grupo ludar también distingue subgrupos de acuerdo al lugar desde el cual emigraron. Así, mencionan como grupos distintos a los "bosniacos", los "serbianes", etc.

1.3 La lengua: Los gitanos rrom de Argentina llaman a su lengua rromanés; los gitanos ludar la llaman ludar, limba luderiaste o rumano. Ludar es la denominación más frecuente. La evidencia de que el grupo ludar y el grupo rrom hablan dos lenguas distintas -y no meros dialectos de una misma lengua- proviene no sólo del nombre que éstos les dan, sino también de la imposibilidad de mutua comprensión cuando las hablan (la interacción entre ambos grupos se produce sólo en castellano).

En 1997, González presentó su tesis de Magíster “Descripción fonológica de los gitanos rom rusos de Argentina". En esta presentación señala:

El grupo mayoritario de los gitanos radicados en Argentina proviene de Rusia, aunque también hay un grupo importante al que llaman "greco", y un pequeño grupo que llegó de España.

La situación económica de los gitanos que viven en Argentina cambia radicalmente respecto de los gitanos en Chile. En efecto, en Argentina los gitanos tienen un nivel socioeconómico mucho más elevado, aunque la actividad principal sigue siendo la compra de vehículos usados. Otra actividad importante es la fabricación industrial de grandes recipientes de cobre, utilizados, entre otros, por los laboratorios en producción de químicos. Esta última actividad es la que proporciona mayores ganancias y aquellos que se dedican a desarrollarla a gran escala pertenecen al grupo con mayor poder económico dentro de los gitanos.

En el caso de las mujeres, la situación es muy distinta a la de las gitanas de nuestro país, ya que en Argentina prácticamente no ven la suerte. $\mathrm{Su}$ actividad se limita a ser dueña de casa.

El nomadismo entre los romá argentinos es muy escaso, casi en su totalidad están establecidos, los pocos que aún tienen la costumbre de salir en carpas se encuentran en la zona de Neuquén. En la zona de Buenos Aires no encontramos ninguna familia que saliera en carpa, incluso personas entre 50 y 60 años nunca habían vivido en carpa. 
La lengua: los gitanos de Argentina llaman a su lengua romanés. Para marcar la diferencia del romané de Chile -diferencia de la cual, tanto los romá de Chile como los de Argentina tienen conciencia- los gitanos de Argentina la denominan más específicamente "leasi".

En Argentina, los romá son hablantes nativos de dicha lengua y bilingües romanés-español. El nivel de fidelidad hacia su lengua materna es alto, y no encontramos gitanos que no hablaran su lengua y, al igual que en Chile, la comunicación entre sus pares se hace exclusivamente en romanés. (González 1997: 19).

Con respecto al grupo que nos ocupa, los gitanos grecos, Fernández (2009) señala:

Los karderasha griegos llegaron entre 1880 y 1890; fueron los primeros entre los rom kalderash que lo hicieron. Este grupo también incluye algunos kaldereasha servios. A su llegada todos eran nómadas y acostumbraban vender caballos y herramientas para el trabajo agrícola, y trabajaban como caldereros (trabajadores del cobre, específicamente). En los 30, comenzaron a moverse hacia las grandes ciudades y descubrieron nuevas oportunidades en el comercio de automóviles. Esta es su principal actividad hoy en día.

La lengua romaní se mantiene dentro de este grupo a pesar de la introducción de algunos préstamos del español.

Actualmente, estos rom están establecidos en las ciudades más grandes de Argentina. Al principio de su llegada eran ortodoxos, luego se convirtieron en católicos, y en los últimos años han estado participando en el poderoso movimiento evangelista, que nació en Francia en 1961, con sus propios pastores e iglesias.

La cultura de los rom kalderash se ha preservado en gran parte debido al papel que juegan las mujeres dentro y fuera de la comunidad romaní. Su tradicional y colorida forma de vestir es vista como un signo de su orgullo y una marca de su autoestima como mujeres romaní. No existen tentaciones que las impulsen a adoptar las extravagantes modas del momento. 
Los kalderash griegos están bien insertados económicamente en la sociedad. Por ejemplo, muchos de ellos exhiben 50 o 60 vehículos en cada venta al mismo tiempo en sus propias tiendas. Estos rom establecieron una red de trabajo entre ellos a lo largo de todo el país. Uno puede comprar un carro a un kalderash griego en el sur de Argentina y después pagarlo a otro (pariente o socio del vendedor) en la capital, a 3 mil kilómetros del lugar donde el vehículo se compró en primera instancia.

De acuerdo con Salamanca (c.p.), los gitanos grecos son exclusivamente sedentarizados. En general, la situación económica de estos gitanos es elevada. Las casas de los gitanos grecos son muy similares a las de sus pares sedentarizados de Chile y Argentina, pues poseen un amplio living-comedor, y llamativas cortinas y alfombras. Los gitanos grecos varones de Neuquén se dedican básicamente al comercio de vehículos. Las mujeres ya no practican la quiromancia, sino que se dedican a las labores domésticas y a colaborar con los hombres en el comercio de vehículos.

Los gitanos grecos de Neuquén tienen una educación formal precaria. De hecho, la mayoría de ellos no ha terminado los estudios primarios. Por lo mismo, un alto porcentaje de ellos no lee ni escribe con fluidez.

Todos los gitanos grecos de Neuquén son bilingües de rromanésespañol. La primera lengua que adquieren es el rromanés, pues es la lengua de uso cotidiano en sus hogares. El español lo utilizan cuando están en contacto con los "criollos" o cuando interactúan con un gitano ludar.

El número de gitanos residentes en la ciudad de Neuquén se calcula en unos tres mil, aunque no existen cifras oficiales.

\section{Objetivos}

a) Determinar cuáles son los fonos de la lengua hablada por los gitanos grecos de Neuquén y describirlos de acuerdo con parámetros articulatorios.

b) Determinar cuáles son los fonemas segmentales de la lengua hablada por los gitanos grecos de Neuquén y sus respectivas realizaciones alofónicas. 
c) Comparar el inventario de fonemas del rromanés hablado por los gitanos grecos de Neuquén, con el rromanés hablado por los gitanos rusos y el rromané jorajané hablado en Chile.

\section{Marco de Referencia}

Para determinar los fonemas y sus variaciones alofónicas, se aplicaron las reglas propuestas por Pike (1947) y Burquest (2001).

\section{Premisas para el análisis fonémico}

Pike (1947) y Burquest (2001) proponen partir de las siguientes premisas para el análisis fonémico:

a) Un sonido tiende a ser modificado por el sonido adyacente.

Según Burquest (2001): "Sounds tent to be affeccted by the enviroments in which they occur." Y agrega que:

When a sequence of sounds is produced, the sounds do not occur just one after another as discrete units. Rather, they occur in a single wave of sound, with each individual sound making its own contribution, and they tent to blend into one another where they abut. (Burquest 2001:3-4)

Esto es lo que sucede en el rromanés greco con la contoide nasal velar sonora [ $\eta$ l, la cual sólo ocurre ante las velares $[\mathrm{k}],[\mathrm{x}]$ y $[\mathrm{g}]$ :

Por ejemplo:

[mạ.'gau] '(tú) quieres'

b) Los sonidos (también los fonemas) de una lengua presentan tendencia a la simetría.

La serie de consonantes aspiradas (que incluye los fonemas $/ \mathrm{p}^{\mathrm{h}} /$, $/ \mathrm{t}^{\mathrm{h}} / \mathrm{y} / \mathrm{k}^{\mathrm{h}} /$ en la variante que nos ocupa) reflejan esta tendencia. En efecto, cada una de estas consonantes tiene una contraparte oclusiva sorda "pura" (/p/, /t/ y / k/, respectivamente).

c) Series características de sonidos ejercen una presión estructural en la interpretación fonémica de segmentos o series fónicas sospechosos. 
La aplicación de esta premisa nos lleva a interpretar los fonos [w] y [u] como alófonos del fonema semiconsonántico / w /. En efecto, dado que hay fuerte evidencia para postular un fonema semiconsonántico $/ \mathrm{j} /$ con dos realizaciones alofónicas ([j] y [i]), esto ejerce una presión estructural para que sus contrapartes velarizadas $[w]$ y [u] reciban un tratamiento afín.

\section{Metodología}

\section{Recolección del material}

Los datos fueron recogidos por el Dr. Gastón Salamanca durante los años 2004 y 2005. Las grabaciones se realizaron en la casa de los colaboradores gitanos y en las dependencias del Departamento de Misiones Transculturales de la Iglesia Evangélica Dimensión de Dios, ubicada en la ciudad de Neuquén. Esto porque el proyecto general contemplaba la adaptación al rromanés greco, de un Nuevo Testamento publicado en Francia. El material fue grabado en cinta magnetofónica.

\section{Colaboradores}

Cinco gitanos bilingües rromanés greco-español colaboraron con este trabajo. Para la selección de estos informantes se siguieron las sugerencias planteadas por Samarin (1967). Esto, por cierto, no siempre fue posible, porque la comunidad gitana es particularmente hermética y el solo hecho de obtener algunas grabaciones es una contribución en sí misma.

Los colaboradores fueron tres mujeres cuyas edades fluctuaban entre los 20 y 25 años (Perla, Parako y Suyai) y dos hombres, cuyas edades fluctuaban entre los 50 y 55 años (Tito y Sandro).

\section{Transcripción}

Una primera transcripción fonética se realizó con el alfabeto RFE, para hacer comparables nuestros datos con las descripciones del rromanés ruso (González, 1997) y rromané jorajané de Chile (González y Salamanca, 2001). Luego se traspasó todo el material (lista léxica y cuento) a AFI. En todo este proceso pusimos especial cuidado en advertir sonidos que no están en el inventario fónico del español.

\section{Lista léxica}

La lista utilizada contiene 300 ítems, la mayoría de los cuales fueron elicitados también en las investigaciones sobre el rromanés ruso y el rromané joarajané de Chile. 


\section{Resultados}

Luego de aplicar el marco teórico-metodológico descrito, se determinaron los siguientes fonemas segmentales y sus respectivas realizaciones alofónicas.

\section{Fonemas segmentales del rromanés greco}

\subsection{Vocales}

\begin{tabular}{|c|c|c|}
\hline Fonema & Alófonos & Ejemplos \\
\hline$/ \mathrm{i} /$ & $\begin{array}{l}\text { [i] } \\
\text { alta, cerrada, anterior, no redondeada. } \\
\text { [ə] } \\
\text { Media, cerrada, cental, no redondeada. }\end{array}$ & 'Iluvia' [vi'Jin] \\
\hline$/ \mathrm{e} /$ & $\begin{array}{l}\text { [e] } \\
\text { media, cerrada, anterior, no redondeada. } \\
{\left[\begin{array}{l}{[\ominus]} \\
\text { Media, cerrada, cental, no redondeada. }\end{array}\right.}\end{array}$ & $\begin{array}{l}\text { /'eftino/ 'barato' ['ef.ti.no] } \\
\text { /pa'Je/ 'cerca' [pa'Je] [ [pa'Je] }\end{array}$ \\
\hline$/ \mathrm{a} /$ & $\begin{array}{c}\text { [a] } \\
\text { baja, abierta, central, no redondeada. }\end{array}$ & /a'men/ 'nosotros' [a.'men] \\
\hline$/ \mathrm{o} /$ & $\begin{array}{c}{[\mathrm{o}]} \\
\text { media, cerrada, posterior, redondeada. }\end{array}$ & /o'pre/ 'arriba' [o.'pre] \\
\hline$/ \mathrm{u} /$ & $\begin{array}{c}{[\mathrm{u}]} \\
\text { alta, cerrada, posterior, redondeada. }\end{array}$ & /u'dar/ 'puerta' [u.'ठ̃ar] \\
\hline
\end{tabular}

\subsection{Consonantes}

\begin{tabular}{|c|c|c|}
\hline$/ \mathrm{p} /$ & $\begin{array}{c}{[\mathrm{p}]} \\
\text { oclusiva pura, bilabial, sorda. }\end{array}$ & /paj/ 'agua' [pai] \\
\hline$/ \mathrm{b} /$ & $\begin{array}{c}{[\mathrm{b}]} \\
\text { oclusiva pura, bilabial, sonora. }\end{array}$ & /baj/ 'manga' [bai] \\
\hline$/ \mathrm{p}^{\mathrm{h} /}$ & $\begin{array}{c}{\left[\mathrm{p}^{\mathrm{h}}\right]} \\
\text { oclusiva aspirada, bilabial, sorda. }\end{array}$ & $/ \mathrm{p}^{\mathrm{h}} \mathrm{ak} / \mathrm{ala}^{\prime}\left[\mathrm{p}^{\mathrm{h}} \mathrm{ak}\right]$ \\
\hline$/ \mathrm{t} /$ & $\begin{array}{c}{[\mathrm{t}]} \\
\text { oclusiva pura, postdental, sorda. }\end{array}$ & /ta'loj/ 'paladar' [ta.'loi] \\
\hline
\end{tabular}




\begin{tabular}{|c|c|c|}
\hline$/ \mathrm{d} /$ & $\begin{array}{c}{[\mathrm{d}]} \\
\text { oclusiva pura, postdental, sonora. } \\
\text { Ocurre en todo contexto, } \\
\text { excepto entre vocales. } \\
{[ð]} \\
\text { fricativa, postdental, sonora. } \\
\text { Ocurre en contexto intervocálico. }\end{array}$ & /dara'no/ 'asustado' [da.ra.'no] \\
\hline$/ \mathrm{t}^{\mathrm{h} /}$ & $\begin{array}{c}{\left[\mathrm{t}^{\mathrm{h}}\right]} \\
\text { oclusiva aspirada, postdental, sorda. }\end{array}$ & 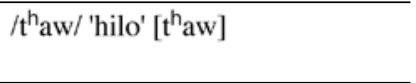 \\
\hline \multirow[t]{2}{*}{$/ \mathrm{k} /$} & $\begin{array}{c}{[\mathrm{k}]} \\
\text { oclusiva pura, velar, sorda. } \\
\text { Ocurre en todo contexto, excepto } \\
\text { ante vocal anterior. } \\
{\left[\mathrm{k}^{\mathrm{j}}\right]}\end{array}$ & /ka'tse/ 'aquí' [ka'.tse] \\
\hline & $\begin{array}{l}\text { oclusiva pura, postpalatal, sorda. } \\
\text { Ocurre ante vocal anterior. }\end{array}$ & /ara'ki/ 'ayer' [a.ra.'kji] \\
\hline \multirow[t]{4}{*}{$/ g /$} & $\begin{array}{c}\mathrm{g}] \\
\text { oclusiva pura, velar, sonora. } \\
\text { Ocurre ante vocal no anterior: } \\
\text { - en posición inicial absoluta; } \\
\text { - tras consonante nasal. }\end{array}$ & $\begin{array}{l}\text { /gad/ 'camisa' [gad] } \\
\text { /3unga'lo/ 'feo' [3un.ga.'lo]. }\end{array}$ \\
\hline & $\begin{array}{c}{\left[\mathrm{g}^{\mathrm{j}}\right]} \\
\text { oclusiva pura, postpalatal, } \\
\text { sonora. Ocurre ante vocal } \\
\text { anterior: } \\
\text { - en posición inicial absoluta; } \\
\text { - tras consonante nasal. }\end{array}$ & $\begin{array}{l}\text { /gel'dala/ 'espejo' [gjel..'da.la] } \\
\text { /man'ges/ 'quieres' [manj.'.gjes] }\end{array}$ \\
\hline & $\begin{array}{c}{[\mathrm{\gamma}]} \\
\text { fricativa, velar, sonora. } \\
\text { Ocurre en contexto intervocálico, } \\
\text { ante vocal no anterior. }\end{array}$ & 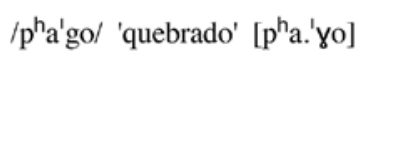 \\
\hline & $\begin{array}{c}{\left[\mathrm{\gamma}^{\mathrm{j}]}\right]} \\
\text { fricativa, postpalatal, sonora. } \\
\text { Ocurre en contexto intervocálico } \\
\text { ante vocal anterior. }\end{array}$ & /go'gi//'cerebro' [go.' ' $\left.{ }^{\mathrm{j}} \mathrm{i}\right]$ \\
\hline \multirow[t]{3}{*}{$/ \mathrm{k}^{\mathrm{h}} /$} & {$\left[\mathrm{k}^{\mathrm{h}}\right]$} & 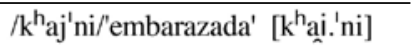 \\
\hline & $\begin{array}{l}\text { oclusiva aspirada, velar, sorda. } \\
\text { Ocurre en todo contexto, excepto } \\
\text { ante vocal anterior. } \\
{\left[\mathrm{k}^{\mathrm{jh}}\right]}\end{array}$ & \\
\hline & $\begin{array}{l}\text { oclusiva aspirada, postpalatal, sorda. } \\
\text { Ocurre ante vocal anterior. }\end{array}$ & $/$ mor' $^{\prime} \mathrm{h}^{\mathrm{h}} \mathrm{i} /$ 'piel' [mor.'k ${ }^{\left.\mathrm{j} \mathrm{h}_{\mathrm{i}}\right]}$ \\
\hline$/ \mathrm{f} /$ & $\begin{array}{c}\text { [f] } \\
\text { fricativa, labiodental, sorda. } \\
{[\phi]} \\
\text { fricativa, bilabial, sorda. } \\
\text { Variación libre entre }[\mathrm{f}] \text { y }[\phi] .\end{array}$ & /'fala/ 'gracias' ['fa.la] ['фa.la] \\
\hline
\end{tabular}




\begin{tabular}{|c|c|c|}
\hline$/ \mathrm{v} /$ & $\begin{array}{l}{[\mathrm{v}]} \\
\text { fricativa, labiodental, sonora. } \\
{[\beta]} \\
\text { fricativa, bilabial, sonora. } \\
\text { Variación libre con }[\mathrm{v}] \text {. }\end{array}$ & /vas/ 'mano' [vas] [ßßas] \\
\hline$/ \mathrm{s} /$ & $\begin{array}{c}{[\mathrm{s}]} \\
\text { fricativa, dorsoalveolar, sorda. }\end{array}$ & /sa'lata/ 'ensalada' [sa.'la.ta] \\
\hline$|z|$ & $\begin{array}{c}{[\mathrm{z}]} \\
\text { fricativa, dorsoalveolar, sonora. }\end{array}$ & /zor/'fuerza' [zor] \\
\hline$/ 5 /$ & $\begin{array}{c}{[S]} \\
\text { Fricativa, alveopalatal, sorda }\end{array}$ & /Saw/ 'hijo' [Saw] \\
\hline$/ 3 /$ & $\begin{array}{c}\text { [3] } \\
\text { Fricativa, alveopalatal, sonora. } \\
\text { [d3] } \\
\text { africada, alveopalatal, } \\
\text { sonora. Variación libre } \\
\text { entre [d3] y [3]. }\end{array}$ & /3an/'vayan!' [3an] [dzan] \\
\hline$/ \mathrm{x} /$ & $\begin{array}{c}\mathrm{x}] \\
\text { fricativa, velar, sorda. }\end{array}$ & /xarnó/ 'corto' [xar.nó] \\
\hline$/ \mathrm{ts} /$ & $\begin{array}{c}\text { [ts] } \\
\text { africada, alveolar, áfona } \\
\end{array}$ & /tsar/ 'muela' [tsar] \\
\hline$/ \mathrm{t} \mathrm{f} /$ & $\begin{array}{c}{[\mathrm{t} f]} \\
\text { africada pura, alveopalatal, sorda. }\end{array}$ & $\begin{array}{l}\text { /'tJaso/ 'reloj' ['tfa.so] } \\
\end{array}$ \\
\hline$/ \mathrm{t}^{\mathrm{h} /}$ & $\begin{array}{l}{\left[\mathrm{t}^{\mathrm{h}}\right]} \\
\text { africada aspirada, alveopalatal, sorda. }\end{array}$ & $/ \mathrm{t} \int^{h_{h}} \mathrm{am} /$ 'mejilla' [t $\int^{h_{\text {am }}}$ \\
\hline$/ \mathrm{m} /$ & $\begin{array}{c}{[\mathrm{m}]} \\
\text { nasal, bilabial, sonora. }\end{array}$ & $/ \mathrm{ma}^{\prime} \mathrm{\int o} /$ 'pescado' [ma.'Jo] \\
\hline \multirow[t]{5}{*}{$/ \mathrm{n} /$} & $\begin{array}{l}\text { [n] } \\
\text { nasal, alveolar, sonora. } \\
\text { Ocurre ante vocal y ente } \\
\text { consonante alveolar. } \\
\text { [n] } \\
\text { nasal, postdental, sonora. } \\
\text { Ocurre ante } \\
\text { consonante postdental. }\end{array}$ & /dan'da/'dientes' [dan.'da] \\
\hline & $\begin{array}{c}{\left[\mathrm{n}^{\mathrm{j}]}\right.} \\
\text { nasal, alveopalatalizada, } \\
\text { sonora. Ocurre ante } \\
\text { consonante alveopalatal. }\end{array}$ & 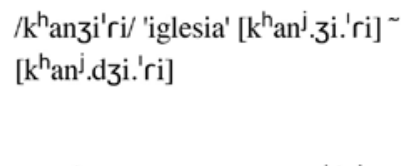 \\
\hline & $\begin{array}{l}\quad\left[\mathrm{p}^{\mathrm{j}}\right] \\
\text { nasal, postpalatal, sonora. } \\
\text { Ocurre ante consonante } \\
\text { postpalatal. }\end{array}$ & /man'ges/ 'quieres' [ manj.'gjes] \\
\hline & $\begin{array}{l}\quad[\mathrm{n}] \\
\text { nasal, velar, sonora. } \\
\text { Ocurre ante consonante } \\
\text { velar. }\end{array}$ & /an'gar/ 'carbón' [ạ.'gar] \\
\hline & $\begin{array}{l}\quad[\mathrm{N}] \\
\text { nasal, uvular, sonora. } \\
\text { Ocurre ante consonante } \\
\text { uvular. }\end{array}$ & /an'во/ 'huevo' [aN. 'во] \\
\hline
\end{tabular}




\begin{tabular}{|c|c|c|}
\hline$/ \mathrm{h} /$ & $\begin{array}{c}{[\mathrm{n}]} \\
\text { nasal, mediopalatal, sonora. }\end{array}$ & /'namo/ 'familia' ['na.mo] \\
\hline$/ 1 /$ & $\begin{array}{c}{[1]} \\
\text { lateral, alveolar, sonora. } \\
{[1]} \\
\text { Lateral, postdental, sonora. } \\
\text { Ocurre con consonante postdental. }\end{array}$ & /'lantso/ 'cadena' ['lan.tso] \\
\hline$/ \mathrm{r} /$ & $\begin{array}{c}{[r]} \\
\text { vibrante simple, alveolar, sonora. }\end{array}$ & /rat/ 'sangre' [rat] \\
\hline$/ \mathrm{B} /$ & $\begin{array}{c}{[\mathrm{b}]} \\
\text { fricativa uvular sonora. } \\
{[\mathrm{R}]} \\
\text { Vibrante múltiple uvular sonora. } \\
\text { [r] } \\
\text { Vibrante múltiple alveolar sonora }\end{array}$ & $\begin{array}{l}\text { /'вatsa/ 'pato' ['ва.tsa], ['Ra.tsa], } \\
\text { ['ra.tsa] }\end{array}$ \\
\hline
\end{tabular}

\subsection{Semiconsonantes}

\begin{tabular}{|c|c|c|}
\hline$/ \mathrm{j} /$ & $\begin{array}{l}\text { [j] } \\
\text { Semiconsonante muy alta, } \\
\text { cerrada, anterior, no } \\
\text { labializada, asilábica. } \\
\text { Ocurre antes de una } \\
\text { vocal tónica. } \\
\text { [i] } \\
\text { Semivocal muy alta, cerrada, } \\
\text { anterior, no labializada, } \\
\text { asilábica. Sigue a una vocal } \\
\text { tónica en la misma sílaba. }\end{array}$ & $\begin{array}{l}\text { /jek/ 'no' [jek] } \\
\text { /bo'jas/ (grupo de gitanos no } \\
\text { /ro'ma/) [bo.'jas] } \\
\text { /Ju'djas/ 'tiró' [Ju'ðjas] } \\
\text { /baj/ 'manga' [bai] }\end{array}$ \\
\hline$/ \mathrm{w} /$ & $\begin{array}{l}{[\mathrm{w}]} \\
\text { Semiconsonante muy alta, } \\
\text { posterior, cerrada, redondeada, } \\
\text { asilábica. Precede a una vocal } \\
\text { tónica. } \\
\quad[u \underline{]}] \\
\text { Semivocal muy alta, } \\
\text { cerrada, posterior, labializada, } \\
\text { asilábica. Sigue a una } \\
\text { vocal tónica. }\end{array}$ & $\begin{array}{l}\text { /'kawit5i/ 'una de las "razas" de } \\
\text { gitanos en Chile' ['ka.wi.t5i] } \\
\text { /a'swin/ 'lágrima' [a.'swin] } \\
\text { /'rodaw/ 'busco' ['ro.ðaun] }\end{array}$ \\
\hline
\end{tabular}




\section{Observaciones respecto de la pronunciación}

a) En algunos informantes, y sólo en determinadas palabras, se producen realizaciones centralizadas [ə] en alternancia libre con [i] y [e].

b) Algunos hablantes no emplean el fonema africado alveolar áfono oral /ts/. En las palabras correspondientes, éste es reemplazado por el fonema africado alveopalatal áfono oral $/ \mathrm{t} \mathrm{J} /$.

c) En algunos hablantes, se produce una fluctuación de fonemas entre /r/ y /r/, como en /'rodaw/ /'rodaw/.

d) En algunos informantes se percibe una pérdida de las consonantes aspiradas.

e) Hay neutralización del contraste entre las oclusivas sonoras y las sordas, y entre las vibrantes, en posición final.

\section{Texto en transcripción fonémica ${ }^{2}$}

A continuación presentamos un cuento relatado por una gitana greca (Perla C.) transcrito fonémicamente. En la columna de la derecha se incluye una traducción semilibre del mismo.

2 El mismo texto, en transcripción fonética, se contiene en Anexos. 
/sas jek ampo'rato $\rightarrow$ I

sas les jek Jaw tsino'во \ا

aj sas jek bom kaj ve'Selas 'ande

'tsera $\rightarrow$ ।

aj ljas 'lesk ${ }_{\text {i }}$ tom'li ge'lis te anel jek

$\mathrm{k}^{\mathrm{h}} \mathrm{o}^{\prime}$ ro le pa'jesko $\searrow 1$

aj ljas o tsino'во le ampora'tosko \।

Su'djas le ba'resa 'ando $\mathrm{k}^{\mathrm{h}} \mathrm{o}^{\text {'ro kaj sas }}$

pe 'lako 'kitjo $\rightarrow$ ।

aj $\mathrm{p}^{\mathrm{h}}$ aga'dja les $\searrow \|$

e вom'li xo'ljajlis \ا

aj djas les ambraj'a $\rightarrow$ ।

$\mathrm{p}^{\mathrm{h}} \mathrm{en}^{\prime} \mathrm{djas} \rightarrow \mathrm{l}$

te del tu o Del $\rightarrow$ ।

'kana va'rjosa $\rightarrow$ ।

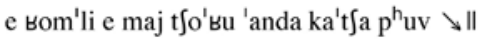

na'k $k^{\text {hlo but }}$ vof $\rightarrow$ ।

aj avi'lis e 'brjama te ansu'rilpe \l

ai $\mathrm{p}^{\mathrm{h}}$ enél lésko dad o amporáto $\rightarrow \mathrm{I}$

'Sava $\rightarrow$ I

avi'lis ki 'brjama te ansu'ristu \l

sa'vi ra'k ${ }^{\mathrm{h}}$ li man'ges $\nearrow$ I

o ra' $k^{\text {h } l o ~ p^{h} e^{\prime} d j a s ~} \rightarrow$ ।

'brea $\rightarrow$ ।

mi sim di'no am'bran ka'tar e som'li $\rightarrow \mid$

aj si te 'rodaw la ra' $k^{\text {h }} \mathrm{lja} \rightarrow$ ।

la maj tfo'tra te ansu'ri man 'lasa $\searrow ॥$

lel aj zal o ra' $k^{\mathrm{h}}$ lo te 'rodel la ra' $\mathrm{k}^{\mathrm{h}} \mathrm{lja} \rightarrow$

\section{Había un rey}

y había un niño pequeño;

y había un gitano que se sentaba en la carpa,

y tomó a su mujer y fue a traer un

balde de agua;

y el hijo del rey agarró (una piedra),

tiró la piedra a la damajuana que estaba

sobre la cintura de la gitana

y la rompió.

La gitana se enojó,

y lo maldijo,

dijo:

te dé Dios,

cuando crezcas,

la mujer más pobre de esta tierra.

Pasó mucho tiempo,

y llegó el tiempo de casarse,

y le dice su padre, el rey:

hijo,

llegó el tiempo de casarte,

¿Qué muchacha quieres?

El hijo dijo:

Padre,

yo estoy maldecido por una gitana

y tengo que buscar a la chica

más pobre para casarme con ella.

Va el chico a buscar a la muchacha 


\begin{tabular}{|c|c|}
\hline la maj t〕o'ва \l & más pobre, \\
\hline aj na' $k^{h}$ los pe jek 'podo $\searrow$ I & y pasó por un puente; \\
\hline afun'djas ke 'varekon pe'los 'ando paj $\downarrow$ I & y escuchó que alguien se cayó en el agua \\
\hline aj tfipisar'dja $\rightarrow$ I & y (él) gritó: \\
\hline kon si ko'the & ¿quién está ahí? \\
\hline djas an'glal jek ra'k $k^{h} l i ~ \rightarrow \mid$ aj $p^{h}$ en'djas $\rightarrow \mid$ & contestó una chica: \\
\hline me $\searrow 1$ & yo; \\
\hline o ra' $k^{h} l o$ di' $k^{h}$ jas la ra' $k^{h} l j a ~ \rightarrow I$ & el chico la vio \\
\hline aj p phe'lja la $\rightarrow$ I & y le preguntó: \\
\hline sar vu'Ses tu $\nearrow$ I & ¿cómo te llamas? \\
\hline voj $\mathrm{p}^{\mathrm{h}}$ en'djas 'leske $\rightarrow$ I & Ella le respondió: \\
\hline 'klara $\rightarrow$ । & Clara. \\
\hline vo $\mathrm{p}^{\mathrm{h}} \mathrm{en}^{\prime} \mathrm{djas} \rightarrow \mathrm{I}$ & Él le dijo: \\
\hline 'klara $\rightarrow$ I & Clara \\
\hline so ke'res 'ando paj $\nearrow$ & ¿Qué haces en el agua? \\
\hline ve' $\int a w$ 'ando paj dege'se $\rightarrow$ I & Vivo en el agua de día, \\
\hline ke naj man ga'da $\rightarrow \mid$ & porque no tengo ropa; \\
\hline aj de rja'te so'vaw te'la o 'podo $\searrow$ l & y de noche duermo debajo del puente. \\
\hline $\mathrm{p}^{\mathrm{h}}$ en'djas 'peske o ra' $\mathrm{k}^{\mathrm{h}} \mathrm{lo}$ 'ando 'gundo $\rightarrow \mid$ & El chico dijo para sí: \\
\hline 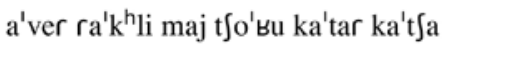 & otra chica más pobre que esta \\
\hline Si ma ara'k $k^{h}$ aw $\rightarrow I$ & no voy a encontrar, \\
\hline aj $\mathrm{p}^{\mathrm{h} e n ' d j a s}$ 'la'ke $\rightarrow$ I & y le dijo: \\
\hline 'klara $\rightarrow$ I & Clara, \\
\hline azu'ker man $\rightarrow$ I & espérame, \\
\hline ke aka'na a'vaw $\rightarrow$ I & que ahora vengo; \\
\hline zaw te ki'naw 'tuke 'gada $\rightarrow$ I & Voy a comprarte ropa \\
\hline aj man'gaw te ansu'ri man 'tusa $\searrow \|$ & y quiero casarme contigo. \\
\hline ljas o ra'k $k^{h} l o \rightarrow l$ & El chico \\
\hline an'djas 'lake ga'da $\rightarrow$ । & le trajo ropa \\
\hline aj ninger'djas la kaj 'pesko dad & y la llevó a su padre \\
\hline
\end{tabular}




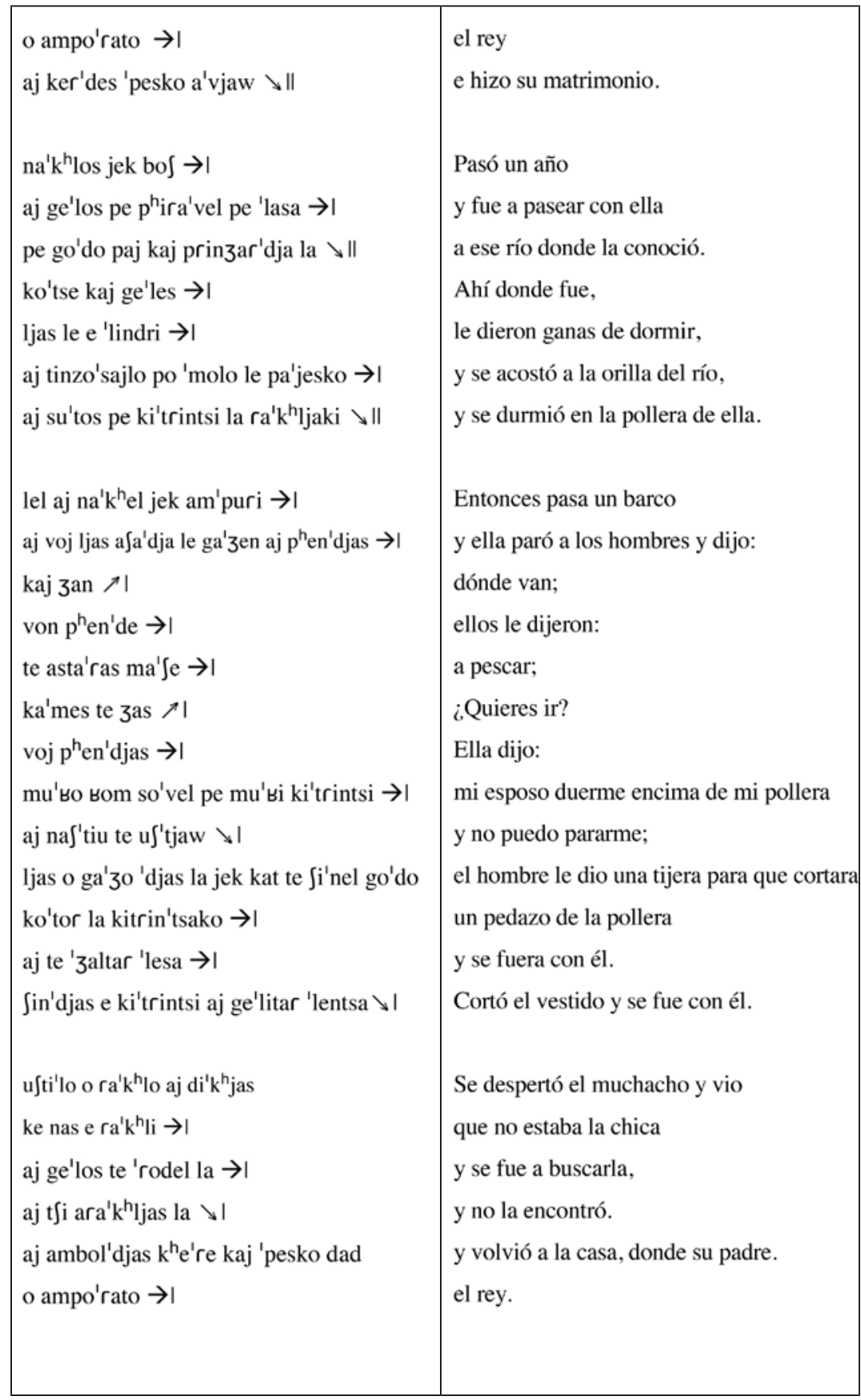


aj puf'ljas 'lesko dad $\rightarrow$ ।

kaj e 'klara ki tom'li

$\mathrm{p}^{\mathrm{h}} \mathrm{en}^{\prime}$ djas o ra' $\mathrm{k}^{\mathrm{h}} \mathrm{lo} \rightarrow \mathrm{I}$

t $\int i$ za'naw $\rightarrow \mid$

ke afi'lem su'to pe 'laki ki'trintsi $\rightarrow$ ।

aj 'kana ufti'lem $\nearrow$ I

ara'k ${ }^{\mathrm{h}}$ lem ka'ko ko'tor la kitrin'tsako $\rightarrow$ ।

ro'dem la $\rightarrow$ ।

aj t $\int \mathrm{i}$ ara' $k^{\mathrm{h}} \mathrm{lem}$ la $\searrow \|$

na' $k^{\mathrm{h}}$ los but gje'sa $\rightarrow$ ।

aj vo ro'djala pe sa le 'foruja $\rightarrow$ ।

ljas aj ge'los 'ande jek 'foro $\rightarrow$ ।

aj sas jek pa'kiuv le ga'zengi kaj asta'ren ma'se \l

a'venas le ga'ze 'peske ga'zantsa $\rightarrow$ I aj avi'los jek ga'zo 'peska ga'zasa $\rightarrow$ । go'dja ga'zi sas e 'klara 'leski ga'zi $\rightarrow$ ।

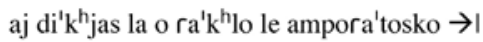

aj prinzar'djas la $\rightarrow$ ।

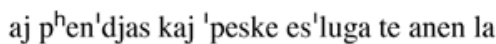
ra'k $k^{h} l j a$ 'leste $\searrow$ I

$\mathrm{p}^{\mathrm{h}} \mathrm{en}^{\prime}$ djas 'leske e ra' $\mathrm{k}^{\mathrm{h}} \mathrm{li} \rightarrow \mathrm{I}$

ko san tu $\nearrow$ I

so man'ges $\lambda$

vo $\mathrm{p}^{\mathrm{h}} \mathrm{en}^{\prime} \mathrm{dj}$ as $\rightarrow$ ।

tfi se'res 'tuke 'mandar 7 ।

me sem go'do ze'no kaj ansuri'sajlos 'tusa

$\mathrm{p}^{\mathrm{h}}$ en'djas kaj 'peske es'luga te a'nen

jek 'vurjo $\mathrm{p}^{\mathrm{h}} \mathrm{er}$ 'do paj $\downarrow$ I

aj $t^{\mathrm{h}} \mathrm{o}^{\prime}$ djas la an'dre $\searrow$ I
Y le preguntó su padre:

¿Dónde está Clara, tu esposa?

Dijo el muchacho:

no sé;

me quedé dormido en su pollera

y cuando me levanté,

encontré este pedazo de pollera;

la busqué,

y no la encontré.

Pasaron muchos días

y la buscaba por todos los pueblos,

y se fue a una ciudad

donde había una fiesta de unos pescadores

venían los hombres con sus esposas,

y vino un hombre con su esposa

(esa mujer era Clara, su mujer).

Y la vio el hijo del rey,

y la reconoció;

y le dijo a sus sirvientes que trajeran

a la muchacha donde él.

Le dijo la muchacha a él:

¿Quién eres tú?

¿Qué quieres?

Él le dijo:

¿No te acuerdas de mí?

Yo soy ese hombre que se casó contigo.

Dijo a sus sirvientes que trajeran

un barril lleno de agua,

y la puso adentro. 


\begin{tabular}{|c|c|}
\hline voj $\mathrm{p}^{\mathrm{h}} \mathrm{en}^{\prime}$ djas $\rightarrow \mathrm{I}$ & Ella dijo: \\
\hline 'soske ke'res man'ge ga'dja & ¿Por qué haces esto? \\
\hline vo $\mathrm{p}^{\mathrm{h}} \mathrm{en}^{\prime}$ djas la'ke $\rightarrow$ । & Él le dijo: \\
\hline 'ando paj lem tu $\searrow \mid$ & del agua te saqué, \\
\hline 'ando paj me 'kaw tu \l & en el agua te dejaré. \\
\hline aj después ro'djas 'peske jek ra'k $\mathrm{k}^{\mathrm{h}} \mathrm{li} \rightarrow \mid$ & Y después encontró otra muchacha, \\
\hline aj ansuri'sajlo 'lasa $\rightarrow \mid$ & y se casó con ella; \\
\hline voj u'lila les but $\rightarrow \mid$ & ella lo amaba \\
\hline aj vo sas varika'no 'lasa $\rightarrow \| /$ & y él estaba feliz con ella. \\
\hline
\end{tabular}




\section{Nota comparativa}

Presentamos una tabla comparativa con los fonemas y alófonos del rromané de Chile, rromanés ruso y rromanés greco a fin de observar las diferencias más prominentes entre estas tres variantes ${ }^{3}$.

\begin{tabular}{|c|c|c|c|c|c|}
\hline \multicolumn{2}{|c|}{ Rromané de Chile } & \multicolumn{2}{|c|}{ Rromanés greco } & \multicolumn{2}{|c|}{ Rromanés ruso } \\
\hline Vocales & & Vocales & & Vocales & \\
\hline Fonemas & Alófonos & Fonemas & Alófonos & Fonemas & Alófonos \\
\hline /i/ & [i] & /i/ & $\begin{array}{l}{[\mathrm{i}]} \\
{[ə]}\end{array}$ & /i/ & [i] \\
\hline /e/ & $\begin{array}{l}{[\mathrm{e}]} \\
{[\varepsilon]}\end{array}$ & /e/ & $\begin{array}{l}{[\mathrm{e}]} \\
{[ə]}\end{array}$ & /e/ & [e] \\
\hline$/ a /$ & [a] & $/ a /$ & [a] & $/ a /$ & [a] \\
\hline$/ 0 /$ & [o] & $/ \mathrm{o} /$ & [o] & $/ \mathrm{o} /$ & [o] \\
\hline$/ \mathrm{u} /$ & {$[u]$} & $/ \mathrm{u} /$ & {$[u]$} & $/ \mathrm{u} /$ & {$[\mathrm{u}]$} \\
\hline Consonan & & Consonan & & Consonar & \\
\hline Fonemas & Alófonos & Fonemas & Alófonos & Fonemas & Alófonos \\
\hline$/ \mathrm{p} /$ & [p] & $/ \mathrm{p} /$ & [p] & $/ \mathrm{p} /$ & [p] \\
\hline$/ \mathrm{p}^{\mathrm{h} /}$ & {$\left[\mathrm{p}^{\mathrm{h}}\right]$} & $/ \mathrm{p}^{\mathrm{h} /}$ & {$\left[\mathrm{p}^{\mathrm{h}}\right]$} & $/ \mathrm{p}^{\mathrm{h} /}$ & {$\left[\mathrm{p}^{\mathrm{h}}\right]$} \\
\hline$/ \mathrm{b} /$ & [b] & $/ \mathrm{b} /$ & [b] & $/ \mathrm{b} /$ & [b] \\
\hline$/ \mathrm{t} /$ & {$[t]$} & $/ \mathrm{t} /$ & {$[t]$} & $/ \mathrm{t} /$ & {$[\mathrm{t}]$} \\
\hline$/ \mathrm{t}^{\mathrm{h}} /$ & {$\left[\mathrm{t}^{\mathrm{h}}\right]$} & $/ \mathrm{t}^{\mathrm{h}} /$ & {$\left[\mathrm{t}^{\mathrm{h}}\right]$} & $/ \mathrm{t}^{\mathrm{h} /}$ & {$\left[\mathrm{t}^{\mathrm{h}}\right]$} \\
\hline$/ \mathrm{d} /$ & $\begin{array}{l}\text { [d] } \\
\text { [ð] }\end{array}$ & $/ \mathrm{d} /$ & $\begin{array}{l}\text { [d] } \\
\text { [ठ] }\end{array}$ & $/ \mathrm{d} /$ & $\begin{array}{l}\text { [d] } \\
\text { [ठ] }\end{array}$ \\
\hline
\end{tabular}

3 Estas diferencias son relevadas con un tamaño de letra ligeramente mayor. 


\begin{tabular}{|c|c|c|c|c|c|}
\hline$/ \mathrm{k} /$ & $\begin{array}{l}{\left[\mathrm{k}^{\mathrm{j}}\right]} \\
{[\mathrm{k}]}\end{array}$ & $/ \mathrm{k} /$ & $\begin{array}{l}{\left[\mathrm{k}^{\mathrm{j}}\right]} \\
{[\mathrm{k}]}\end{array}$ & $/ \mathrm{k} /$ & $\begin{array}{l}{\left[\mathrm{k}^{\mathrm{j}}\right]} \\
{[\mathrm{k}]}\end{array}$ \\
\hline$/ \mathrm{k}^{\mathrm{h}} /$ & $\begin{array}{l}{\left[\mathrm{k}^{\mathrm{h}}\right]} \\
{\left[\mathrm{k}^{\mathrm{jh}}\right]}\end{array}$ & $/ \mathrm{k}^{\mathrm{h} /}$ & $\begin{array}{l}{\left[\mathrm{k}^{\mathrm{h}}\right]} \\
{\left[\mathrm{k}^{\mathrm{jh}}\right]}\end{array}$ & $/ \mathrm{k}^{\mathrm{h}} /$ & $\begin{array}{l}{\left[\mathrm{k}^{\mathrm{h}}\right]} \\
{\left[\mathrm{k}^{\mathrm{jh}}\right]}\end{array}$ \\
\hline$/ g /$ & $\begin{array}{l}{[\mathrm{g}]} \\
{\left[\mathrm{g}^{\mathrm{j}}\right]} \\
{[\mathrm{y}]} \\
{\left[\mathrm{\gamma}^{\mathrm{i}}\right]}\end{array}$ & $/ \mathrm{g} /$ & $\begin{array}{l}{[\mathrm{g}]} \\
{\left[\mathrm{g}^{\mathrm{j}}\right]} \\
{[\mathrm{\gamma}]} \\
{\left[\mathrm{\gamma}^{\mathrm{i}}\right]}\end{array}$ & $/ \mathrm{g} /$ & $\begin{array}{l}{[\mathrm{g}]} \\
{\left[\mathrm{g}^{\mathrm{j}}\right]} \\
{[\mathrm{\gamma}]} \\
{\left[\mathrm{\gamma}^{\mathrm{i}}\right]}\end{array}$ \\
\hline /f/ & $\begin{array}{l}{[\mathrm{f}]} \\
{[\phi]}\end{array}$ & $/ \mathrm{f} /$ & $\begin{array}{l}{[\mathrm{f}]} \\
{[\phi]}\end{array}$ & $/ \mathrm{f} /$ & $\begin{array}{l}{[\mathrm{f}]} \\
{[\phi]}\end{array}$ \\
\hline$/ \mathrm{v} /$ & $\begin{array}{l}{[\mathrm{v}]} \\
{[\beta]}\end{array}$ & $/ \mathrm{v} /$ & $\begin{array}{l}{[\mathrm{v}]} \\
{[\beta]}\end{array}$ & $/ \mathrm{v} /$ & $\begin{array}{l}{[\mathrm{v}]} \\
{[\beta]}\end{array}$ \\
\hline$/ \mathrm{s} /$ & {$[\mathrm{s}]$} & $/ \mathrm{s} /$ & {$[\mathrm{s}]$} & $/ \mathrm{s} /$ & {$[\mathrm{s}]$} \\
\hline$|z|$ & [z] & $\begin{array}{l}\mid z / \\
/ S /\end{array}$ & $\begin{array}{l}{[\mathrm{z}]} \\
{[S]}\end{array}$ & $\begin{array}{l}\mid z / \\
/ S /\end{array}$ & $\begin{array}{l}{[\mathrm{z}]} \\
{[S]}\end{array}$ \\
\hline & & $|3|$ & $\begin{array}{l}{[3]} \\
{[\mathrm{d} 3]}\end{array}$ & $/ 3 /$ & [3] \\
\hline $\mid \mathrm{x} /$ & {$[\mathrm{x}]$} & $/ \mathrm{x} /$ & {$[\mathrm{x}]$} & $\mid \mathrm{x} /$ & {$[\mathrm{x}]$} \\
\hline
\end{tabular}




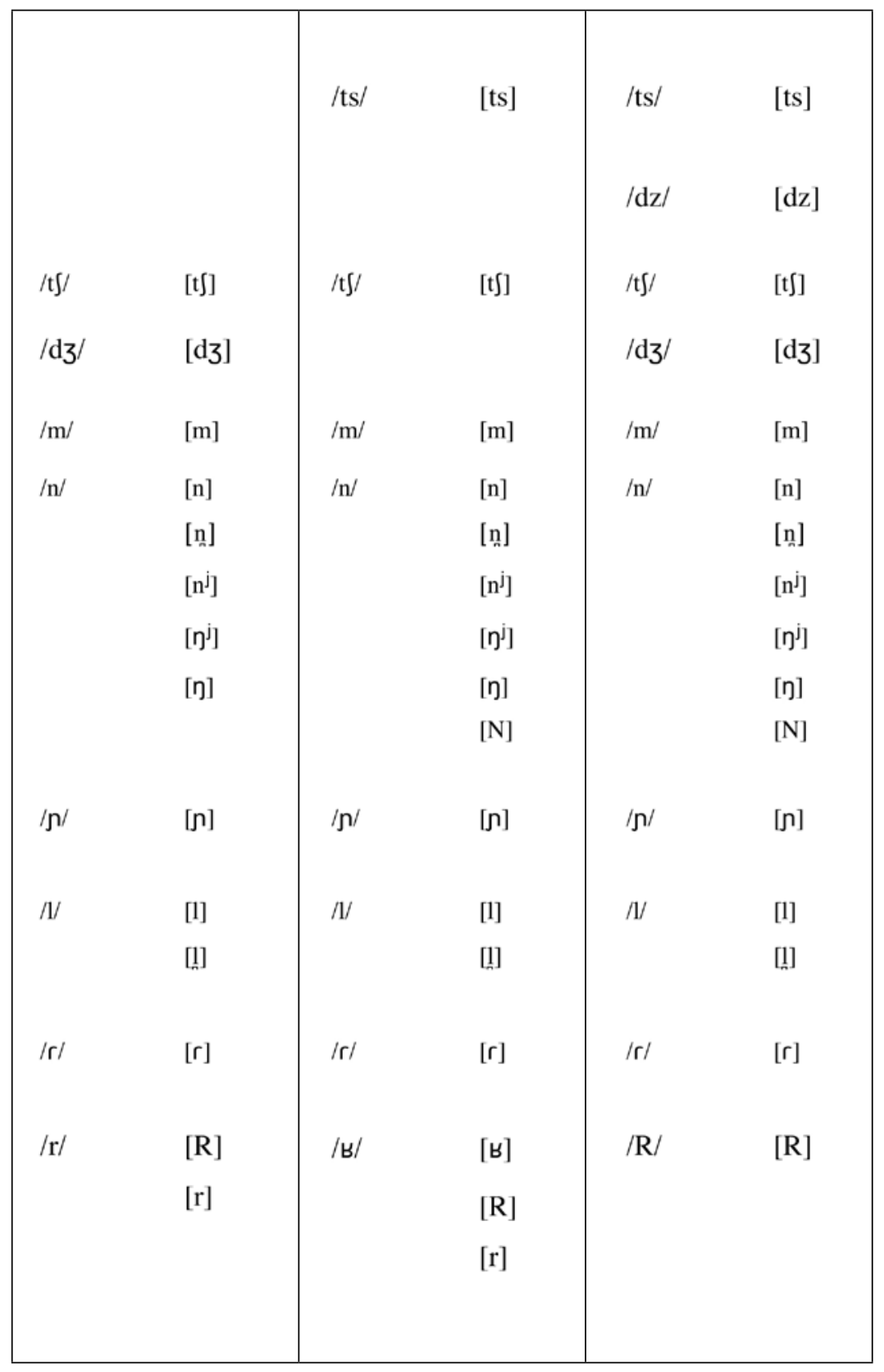




\begin{tabular}{|c|c|c|c|c|c|}
\hline \multicolumn{2}{|c|}{ Semiconsonantes } & \multicolumn{2}{|c|}{ Semiconsonantes } & \multicolumn{2}{|c|}{ Semiconsoantes } \\
\hline Fonemas & Alófonos & Fonemas & Alófonos & Fonemas & Alófonos \\
\hline \multirow[t]{2}{*}{$/ \mathrm{j} /$} & [j] & $/ \mathrm{j} /$ & [j] & $/ \mathrm{j} /$ & [j] \\
\hline & {$[\mathrm{i}]$} & & [i ] & & [i ] \\
\hline \multirow[t]{2}{*}{$/ \mathrm{w} /$} & [w] & $/ \mathrm{w} /$ & [w] & $/ \mathrm{w} /$ & [w] \\
\hline & [u] & & [u] & & {$[\mathrm{u}]$} \\
\hline
\end{tabular}

\subsection{Comentarios}

Como se observa, básicamente hay simetrías entre los sistemas fonético-fonológicos presentados, lo que no es de extrañar, pues se trata de la misma lengua. Sin embargo, así como hay diferencias en el nombre que le dan a sus respectivas variantes (rromané jorajané, en Chile; rromanés ruso, para una variante de Buenos Aires; y rromanés greco, para la que nos ocupa), hay algunas asimetrías en dichos inventarios.

En el plano vocálico, por ejemplo, destaca el hecho de que comparten el mismo inventario de fonemas; pero en las realizaciones alofónicas, se releva que la variante centralizada [ə] sólo se presenta en el rromanés greco.

En el plano consonántico, destaca que el rromanés greco tiene los fonemas $/ \mathrm{S} /, / 3 / \mathrm{y} / \mathrm{ts} /$, que no se presentan en rromané jorajané de Chile, pero sí en el rromanés ruso. Ahora, con respecto a este último, difiere en que no tiene como fonemas los segmentos $/ \mathrm{dz} / \mathrm{y} / \mathrm{dz} /$.

En cuanto a las realizaciones alofónicas consonánticas, se observa que en el rromanés greco hay tres segmentos que son realizaciones del mismo fonema $([\mathrm{B}],[\mathrm{R}] \mathrm{y}[\mathrm{r}])$; en cambio en el rromané jorajané sólo dos $([\mathrm{R}] \mathrm{y}$ [r]) y en el rromanés ruso, sólo uno ([R]). La frecuencia mayoritaria de la variante fricativa uvular [ $\mathrm{b}$ ] en el rromanés greco, a diferencia de las otras variantes, lleva a escogerla como la forma básica. 
Cabe destacar que, en algunos casos, la presencia de determinadas realizaciones se explica bien por el contacto. Por ejemplo, la alta frecuencia del fono [3] -que lleva a interpretarlo como la forma básica del fonema (y no [dz])- se puede explicar por su presencia recurrente en el español de Argentina. Otras explicaciones para las diferencias merecen un tratamiento exhaustivo que no constituía uno de los objetivos de esta tesis.

\section{Consideraciones Finales}

Más que conclusiones, a la manera como se estila en investigaciones de carácter experimental, en este apartado final, resumimos algunos aspectos prominentes que emergen al considerar el inventario de fonemas y alófonos del rromanés greco y su comparación con las variantes rromané jorajané de Chile y rromanés ruso, de Buenos Aires.

Así, como se observa en los resultados, existe una alta simetría entre los sistemas fonético-fonológicos revisados. A pesar de esto, existen algunas diferencias.

En el plano vocálico, la asimetría se observa en los fonemas /i/, /e/, pues, en el rromané jorajané, el fonema /e/ presenta dos variaciones alofónicas ([e] y [ع]); en el rromanés ruso sólo uno ([e]); y en el rromanés greco, dos: [e] y [ə]. En el caso del fonema vocálico / i / , el rromanés greco presenta una diferencia importante respecto de las otras dos variantes, ya que presenta dos variaciones alofónicas: [i] y [ə], a diferencia de las variantes jorajanés y rusa que sólo presentan el alófono [i].

En el plano consonántico, por su parte, se observa que las asimetrías se presentan en los modos de articulación fricativo, africado y vibrante. Estas se observan en mayor cantidad en la variante jorajané. En efecto:

a) El fonema / $\int /$ se presenta con un alófono [ $]$ ] en las variantes rusa y greca; en el caso de la variante jorajané, dicho fonema no se presenta.

b) El fonema / 3 / se presenta con dos alófonos en la variante greca: [3] y [d3]; en la variante rusa, sólo presenta un alófono [3]; mientras que en la variante jorajané no se presenta. 
c) El fonema / dz/ se presenta en la variante rusa y jorajané con un alófono [dz]. En la variante greca, en cambio, ocurre como alófono, pero no es forma básica de ningún fonema.

d) El fonema / ts / se presenta con un alófono [ts] en las variantes rusa y greca; en tanto que la variante jorajané no se presenta.

e) Es en el eje de las vibrantes donde se observa la mayor asimetría. En efecto, en el caso de la variante jorajané, el fonema / r / presenta dos alófonos: $[\mathrm{R}]$ y $[\mathrm{r}$ ]; en la variante greca, el fonema / $\mathrm{B} /$ presenta los alófonos $[\mathrm{B}][\mathrm{r}]$ y $[\mathrm{R}]$; mientras que en la variante rusa, el fonema / R/ presenta sólo un alófono: $[R]$.

Otras consideraciones que se relevan en esta variante y las proyecciones de este trabajo en su totalidad las señalaremos al finalizar nuestra segunda presentación.

\section{Referencias}

Burquest, D. 2001. Phonological Analysis: A functional approach. Dallas: Summer Institute of Linguistics.

Fernández, J. 2009. "Los Rom en las Américas". En el sitio Gypsy World (http: / / www.mundogitano.net/index.php / multimedia / documentos / 116-los-rom-en-lasamericas-fernandez-bernal.html).

González, A. 1997. Descripción fonológica de los rom rusos de Argentina. Tesis para optar al grado de Magíster en Lingüística. Universidad de Concepción.

González A. \& Salamanca, G. 2001. “Descripción fonológica del romané de Chile". Revista de Filología y Lingüística de la Universidad de Costa Rica, XXVII, 177-197.

Pike, K. 1947. Phonemics: A Technique for Reducing Languages to Writing. Ann Arbor: The University of Michigan Press.

Salamanca, G. 1997. Descripción fonológica del ludar, lengua de un grupo de gitanos de Argentina. Tesis de Magíster en Lingüística. Universidad de Concepción. 
Salamanca, G. \& González, A. 1999. “Gitanos de Chile: Un acercamiento etnolingüístico". Atenea 480, 141-172.

Salamanca, G. \& Lizarralde, D. 2008. Propuesta de un grafemario para el rromané jorajané, lengua hablada por los gitanos de Chile (1). Universum, 23(1), 226-247.

Samarin, W. 1967. Field Linguistics. New York: Holt, Rinehard and Wiston, Inc.

\section{Anexos}

\section{Cuento en transcripción fonética}

[sas jek ampo'rato $\rightarrow \mid$ ai sas les jek Jaw tsino'во $\searrow$ I sas jek bom kai ve'Jelas

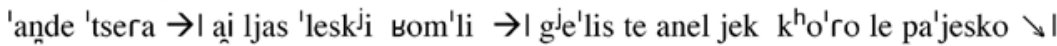

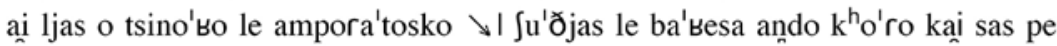
'lako 'kjitjo $\rightarrow$ I ai $\mathrm{p}^{\mathrm{h}}$ aya'ðjales $\searrow \|$

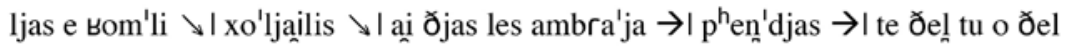

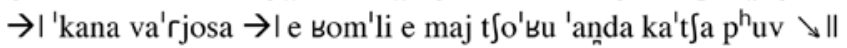

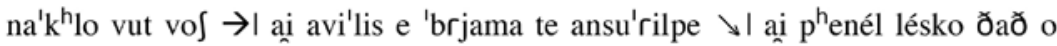

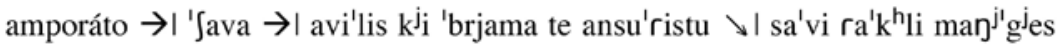

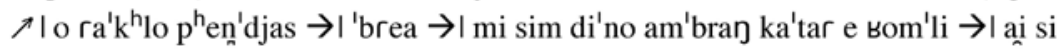

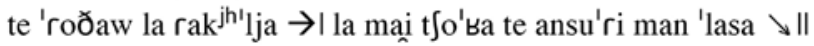

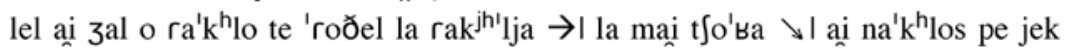

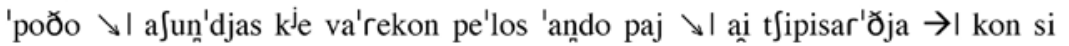

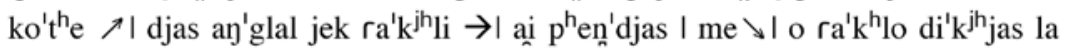

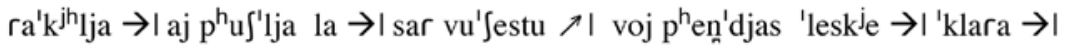

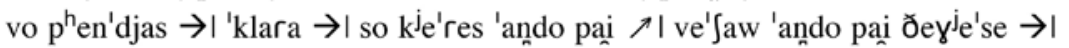

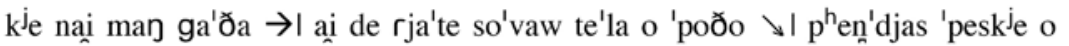

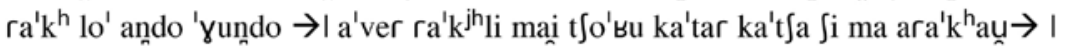
ai $\mathrm{p}^{\mathrm{h}} \mathrm{en}^{\prime}$ djas la'k $\mathrm{k}_{\mathrm{j}} \rightarrow \mid$ 'klara $\rightarrow \mid$ azu'kjer man $\rightarrow \mid \mathrm{k}^{\mathrm{j} e}$ aka'na a'vau $\rightarrow \mid$ zau te

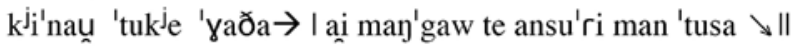

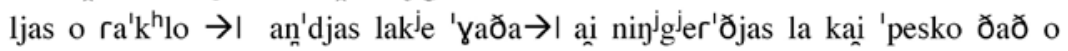
ampo'rato $\rightarrow$ I ai $\mathrm{k}^{\mathrm{j} e r}$ 'ðes 'pesko a'vjau $\searrow \|$

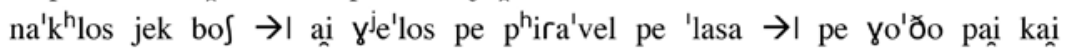

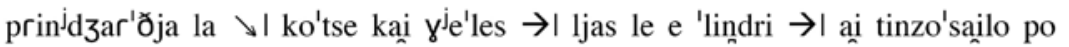

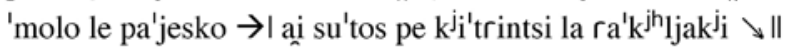




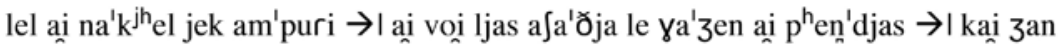

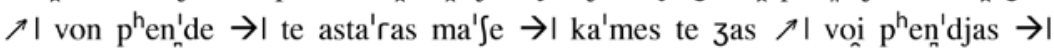

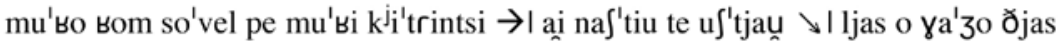
la jek kat te fi'nel yo'бo ko'tor la k'jitrin'tsako $\rightarrow$ I ai te 'zaltar 'lesa $\rightarrow$ I Sin'djas e

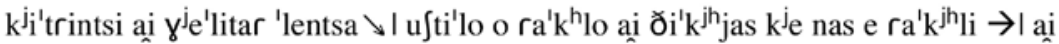

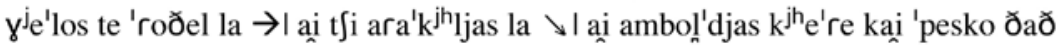

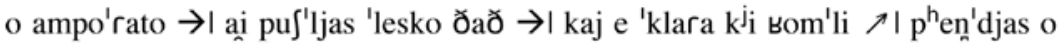

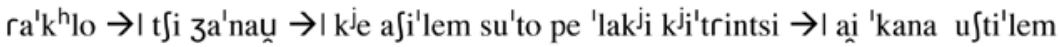
ara' ${ }^{\text {jh }}$ lem ka'ko ko'tor la $k^{j}$ itrin'tsako $\rightarrow \mid$ ro'ðem la $\rightarrow \mid$ ai $t \int i$ ara' $k^{\text {jh }}$ lem la $\searrow \|$ na' $\mathrm{k}^{\mathrm{h}} \mathrm{los}$ but gjje'sa $\rightarrow \mathrm{I}$ ai vo ro'ðjala pe sa le 'foruja $\rightarrow$ l ljas ai $\gamma^{\mathrm{j}} \mathrm{e}^{\mathrm{\prime}}$ los 'ande jek

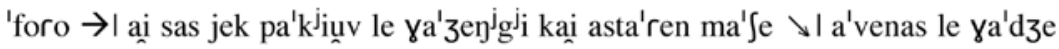
'peskje ya'zantsa $\searrow$ aj avi'los jek ga'zo 'peska ya'zasa $\rightarrow$ go'ðja ya'zi sas e 'klara 'leskji ya'zi $\rightarrow \mid$ ai $\partial^{j} j^{j} k^{j h}$ jas la o ra'k $k^{h} l o$ le ampora'tosko $\rightarrow \mid$ ai

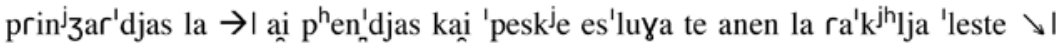

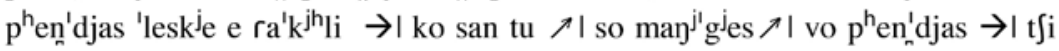
se'res 'tukje 'mandar $\nearrow$ I me sem go'oro ze'no kai ansuri'sailos 'tusa $\searrow \mid p^{h^{h}} n_{n}^{\prime}$ 'djas

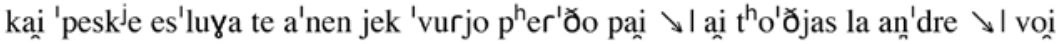

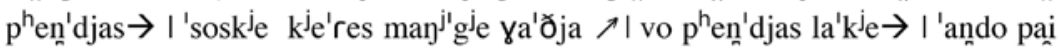
lem tu $\rightarrow$ I 'ando pai me 'kauntu $\searrow$ I ai después ro'ðjas 'peske jek ra'khli $\rightarrow$ a ai ansuri'sailo 'lasa $\rightarrow$ | voi u'lila les but $\rightarrow$ | ai vo sas varika'no 'lasa $\rightarrow$ || ] 